\title{
The Dense Filamentary Giant Molecular Cloud G23.0-0.4: Birthplace of Ongoing Massive Star Formation
}

\author{
Yang $\mathrm{Su}^{1,2}$, Shaobo Zhang ${ }^{1,2}$, Xiangjun Shao ${ }^{1,3}$, and Ji Yang ${ }^{1,2}$ \\ 1 Purple Mountain Observatory, Chinese Academy of Sciences, Nanjing 210008, China \\ ${ }^{2}$ Key Laboratory of Radio Astronomy, Chinese Academy of Sciences, Nanjing 210008, \\ China \\ ${ }^{3}$ Graduate University of the Chinese Academy of Sciences, 19A Yuquan Road, Shijingshan \\ District, Beijing 100049, China
}

\begin{abstract}
We present observations of 1.5 square degree maps of the ${ }^{12} \mathrm{CO},{ }^{13} \mathrm{CO}$, and $\mathrm{C}^{18} \mathrm{O}(J=1-0)$ emission toward the complex region of the supernova remnant (SNR) W41 and SNR G22.7-0.2. A massive $\left(\sim 5 \times 10^{5} M_{\odot}\right)$, large $(\sim 84 \times 15 \mathrm{pc})$, and dense $\left(\sim 10^{3} \mathrm{~cm}^{-3}\right)$ giant molecular cloud (GMC), G23.0-0.4 with $V_{\mathrm{LSR}} \sim 77 \mathrm{~km} \mathrm{~s}^{-1}$, is found to be adjacent to the two SNRs. The GMC displays a filamentary structure approximately along the Galactic plane. The filamentary structure of the dense molecular gas, traced by $\mathrm{C}^{18} \mathrm{O}(J=1-0)$ emission, is also coincident well with the distribution of the dust-continuum emission in the direction. Two dense massive MC clumps, two $6.7 \mathrm{GHz}$ methanol masers, and one HII/SNR complex, associated with the $77 \mathrm{~km} \mathrm{~s}^{-1}$ GMC G23.0-0.4, are aligned along the filamentary structure, indicating the star forming activity within the GMC. These sources have periodic projected spacing of $0.18-0.26$ along the giant filament, which is consistent well with the theoretical predictions of 0.22 . It indicates that the turbulence seems to dominate the fragmentation process of the dense gaseous filament on large scale. The established $4.4 \mathrm{kpc}$ distance of the GMC and the long dense filament traced by $\mathrm{C}^{18} \mathrm{O}$ emission, together with the rich massive star formation groups in the nearby region, suggest that G23.0-0.4 is probably located at the near side of the Scutum-Centaurus arm in the first quadrant. Considering the large scale and the elongation structure along the Galactic plane, we speculate that the dense filamentary GMC has relation to the spiral density wave of the Milky Way.
\end{abstract}

Subject headings: ISM: clouds - ISM: individual (GMC G23.0-0.4) - ISM: molecules - stars: formation 


\section{INTRODUCTION}

The molecular gas is mostly within the Galactic plane and the giant molecular clouds (GMCs) are more concentrated toward spiral arms of the Milky Way (Solomon et al. 1985; Stark \& Lee 2006). The GMCs, as tracers of the large-scale structure in the Galaxy, are birthplaces of most of massive stars in the Galactic plane. Although they are rare in the Galaxy, massive stars significantly affect their surroundings (e.g., Walch 2014). Determining the nature of massive star formation is difficult because of its complexities (e.g., the short lifetime, the obscuration by their parent dust clouds, and the many processes therein). Therefore, understanding the properties of the GMCs is a key step toward understanding massive star formation. Moreover, the dense GMCs are also helpful in constructing the largescale Galactic structure since they are a good tracer of the spiral structure in the Milky Way (e.g., Goodman et al. 2014).

André et al. (2014) recently synthesized a comprehensive physical picture to describe star formation in dense cores of filamentary networks of molecular clouds (MCs). Several cases were also studied in observations, e.g., the infrared dark cloud (IRDC) Nessie (Jackson et al. 2010), the Rosette GMC (Schneider et al. 2012), the IRDC G14.225-0.506 (Busquet et al. 2013), the Cygnus OB 7 GMC (Dobashi et al. 2014), and the IRDC G011.11-0.12 (Ragan et al. 2015). Takahashi et al. (2013) summarized the hierarchical fragmentation structure of the Orion Molecular Cloud (OMC) filaments from GMCs scale ( $\approx 35 \mathrm{pc}$ ) to small-scale clumps scale $(\approx 0.3 \mathrm{pc})$. Along the dense filament, massive stars probably form from these dense fragmentational regions (Jackson et al. 2010). However, the fragmentation structures of other long dense GMC filaments are less studied. It is partly due to the complicated morphology of MCs, the superposition of the emission of molecular gas along the line of sight (LOS), and the strong disruption of GMCs by the massive stars feedback within it (Li et al. 2013; Ragan et al. 2014).

On the other hand, it is powerful to combine the dust emission and the molecular line dataset in investigating the nature of the filamentary structure. In the paper, the column density distributions of the densest structures can benefit from the ATLASGAL dust-continuum survey, while the kinematical information about the dense filament can be gotten from our CO and their isotopes survey (see Section 2).

GMC G23.0-0.4, which is centered at $(l, b, v)=\left(23.0,-0.4,77 \mathrm{~km} \mathrm{~s}^{-1}\right)$ and roughly aligned with the Galactic Plane, has a coherent velocity structure over about $1.1 \times 0.2$ as traced by ${ }^{13} \mathrm{CO}(J=1-0)$ and $\mathrm{C}^{18} \mathrm{O}(J=1-0)$ emissions (Figure 2, also see Su et al. 2014). Several H II regions (see the H II region catalogs in Lockman 1989; Lockman et al. 1996; Anderson et al. 2011, 2014), a group of early-type massive stars(Messineo et al. 2014), and two SNRs (Green 2014) are located in the field of view (FOV) of the GMC. The distances 
of most of above objects are consistent with that of GMC G23.0-0.4 (Messineo et al. 2010, 2014; Su et al. 2014). G23.0-0.4, as a long dense GMC, is a good laboratory for the investigation of the properties of the filament. The locally massive stars feedback near/within the dense GMC also give us a good opportunity to study the relationship between them.

In the paper, we mainly focus on the nature of the filamentary GMC G23.0-0.4 in the complicated interstellar medium (ISM). We also discuss the relationship between the GMC and the ambient star forming activity. We present the observations of ${ }^{12} \mathrm{CO}(J=1-0)$, ${ }^{13} \mathrm{CO}(J=1-0)$, and $\mathrm{C}^{18} \mathrm{O}(J=1-0)$ and the data reduction in the following section. Section 3 shows the properties of the GMC. In Section 4, we mainly discuss the fragmentation process along the dense filament. We finally summarize our conclusions in Section 5.

\section{OBSERVATIONS AND DATA REDUCTION}

Observations in the ${ }^{12} \mathrm{CO}(J=1-0),{ }^{13} \mathrm{CO}(J=1-0)$, and $\mathrm{C}^{18} \mathrm{O}(J=1-0)$ were made simultaneously with the 13.7 m millimeter-wavelength telescope, located at Delingha in China. The 9-beam Superconducting Spectroscopic Array Receiver (SSAR) was working as the front end in sideband separation mode (see the details in Shan et al. 2012).

The data were observed using the on-the-fly (OTF) mode, with the standard chopper wheel method for calibration. In the mode, the telescope beam is scanned along lines in the Galactic longitude and latitude directions on the sky at a constant rate of $50^{\prime \prime} \mathrm{s}^{-1}$ and the receiver records spectra every $0.3 \mathrm{sec}$. The data scanned in both longitude and latitude directions were combined together to reduce the fluctuation of noise perpendicular to the scanning direction.

We adopted the main beam efficiency $\eta_{\mathrm{mb}}=0.44$ for ${ }^{12} \mathrm{CO}(J=1-0)$ and 0.48 for ${ }^{13} \mathrm{CO}(J=1-$ $0)$ and $\mathrm{C}^{18} \mathrm{O}(J=1-0)$. The mean rms noise level of the calibrated brightness temperature $\left(T_{\mathrm{R}}\right)$ was $\sim 0.5 \mathrm{~K}$ for ${ }^{12} \mathrm{CO}(J=1-0)$ at a resolution of $0.16 \mathrm{~km} \mathrm{~s}^{-1}$ and $\sim 0.3 \mathrm{~K}$ for ${ }^{13} \mathrm{CO}(J=1-$ $0)$ and $\mathrm{C}^{18} \mathrm{O}(J=1-0)$ at $0.17 \mathrm{~km} \mathrm{~s}^{-1}$. All $\mathrm{CO}$ data were reduced using the GILDAS/CLASS package 1 .

The available CO High Resolution Survey (COHRS, Dempsey et al. 2013) data and the Galactic Ring Survey (GRS, Jackson et al. 2006) data were also used for comparison. The 1.4 GHz VLA Galactic Plane Survey (VGPS, Stil et al. 2006) data and the $870 \mu \mathrm{m}$ ATLASGAL survey (Schuller et al. 2009) data were used to trace the thermal/non-thermal radio emission and the cold dust emission, respectively. We also use the H d data of VGPS to

\footnotetext{
${ }^{1}$ http://www.iram.fr/IRAMFR/GILDAS
} 
resolve the kinematic distance ambiguity of GMC G23.0-0.4 (see Section 4.1). We briefly summarized the information of these survey data in Table 1.

\section{RESULTS}

The molecular gas toward the observational region contains a number of velocity components, which indicates the overlapping MCs along the LOS in the complicated region. It is unsurprising when we consider that a large amount of molecular gas is located in the inner Galaxy. In the direction of $l=23.0$, our LOS will across several spiral arms (the local/OrionCygnus arm, the Carina-Sagittarius arm, the Scutum-Centaurus arm, and the Perseus arm) and the Galactic Bar (e.g., Hammersley et al. 2000; Benjamin et al. 2005).

In Figure 1, we show the typical spectra, which is extracted from a $30^{\prime} \times 30^{\prime}$ region centered at $\left(l=23.25, b=-0^{\circ} 25\right)$. We find that, even for optically thin $\mathrm{C}^{18} \mathrm{O}(J=1-0)$ line, the spectrum is crowded in the velocity interval of $50-110 \mathrm{~km} \mathrm{~s}^{-1}$. Four distinct $\mathrm{C}^{18} \mathrm{O}$ peaks can be discerned at 52.6, 63.6, 77.4, and $96.8 \mathrm{~km} \mathrm{~s}^{-1}$, which probably indicate the different MC components along the LOS. Also, several other MC components (e.g., 57.4, 81.6, and $101.2 \mathrm{~km} \mathrm{~s}^{-1}$ ) can be seen near the above $\mathrm{C}^{18} \mathrm{O}$ peaks.

The complicated MC components in the velocity interval of $50-110 \mathrm{~km} \mathrm{~s}^{-1}$ prevent us to further discuss the relationship between the different MCs. Fortunately, in the velocity range of $70-80 \mathrm{~km} \mathrm{~s}^{-1}$, the $\mathrm{C}^{18} \mathrm{O}(J=1-0)$ emission of GMC G23.0-0.4 that we are interested in suffer less from contamination from other $\mathrm{MC}$ components along the LOS. The filamentary structure of the GMC can be confirmed to be coherent in velocity space, especially from the $\mathrm{C}^{18} \mathrm{O}$ emission (Section 3.1). Moreover, we have shown that the distance of the GMC with LSR velocity of $77 \mathrm{~km} \mathrm{~s}^{-1}$ was determined (see Section 4.1). The established distance allows us to investigate more reliable physical properties of the GMC accordingly. In the section, we mainly study the properties of GMC G23.0-0.4 ( $V_{\mathrm{LSR}} \sim 70-84 \mathrm{~km} \mathrm{~s}^{-1}$; Section 3.1). Other interesting MCs adjacent to the filamentary GMC, which have similar LSR velocity of $\sim 77 \mathrm{~km} \mathrm{~s}^{-1}$, are also investigated (Section 3.2).

\subsection{Dense Filamentary GMC G23.0-0.4}

\subsubsection{Distribution of the CO Emission}

We made the 3-color intensity image in the velocity interval of $V_{\mathrm{LSR}}=74-79 \mathrm{~km} \mathrm{~s}^{-1}$, overlaid on the VGPS $1.4 \mathrm{GHz}$ radio continuum contours (Figure 2). It is clearly seen that 
the overall distribution of the molecular gas is quite complicated in the FOV (Figure 2). We also note that the characteristics of our ${ }^{13} \mathrm{CO}(J=1-0)$ emission are consistent well with that of GRS data. Meanwhile, the $\mathrm{C}^{18} \mathrm{O}(J=1-0)$ emission in our observations can provide further insight into the nature of the dense molecular gas. Here we mainly focus on the brightest filamentary structure: GMC G23.0-0.4.

In the 3-color image, the ${ }^{12} \mathrm{CO}(J=1-0)$ emission in blue is more diffuse and extended than the ${ }^{13} \mathrm{CO}(J=1-0$; green $)$ and $\mathrm{C}^{18} \mathrm{O}\left(J=1-0\right.$; red) emission. In contrast, the $\mathrm{C}^{18} \mathrm{O}(J=1-$ $0)$ emission is only seen in the region of bright ${ }^{13} \mathrm{CO}(J=1-0)$ emission. It is probably because the typical densities traced by them are different (e.g., $\sim 10^{2} \mathrm{~cm}^{-3}$ for optically thick ${ }^{12} \mathrm{CO}(J=1-0), \sim 10^{3} \mathrm{~cm}^{-3}$ for median optical depth ${ }^{13} \mathrm{CO}(J=1-0)$, and $\sim 10^{4} \mathrm{~cm}^{-3}$ for optically thin $\mathrm{C}^{18} \mathrm{O}(J=1-0)$; e.g., Yonekura et al. 2005). We thus expect that the ${ }^{12} \mathrm{CO}(J=1-0)$, ${ }^{13} \mathrm{CO}(J=1-0)$, and $\mathrm{C}^{18} \mathrm{O}(J=1-0)$ emission is from the enveloping layer of low density, the middle layer of intermediate density, and the inner core of high density of a MC, respectively. The $\mathrm{C}^{18} \mathrm{O}(J=1-0)$ emission, which is a good tracer of the high-density molecular gas $\left(\sim 10^{4} \mathrm{~cm}^{-3}\right)$, can reveal the nature of the dense part of a GMC. The blue envelopes, the green intermediate layers, and the red dense cores of the MCs are indeed distinguished in Figure 2. Therefore, it is useful in studying the properties of the GMC by combining the ${ }^{12} \mathrm{CO}(J=1-0),{ }^{13} \mathrm{CO}(J=1-0)$, and $\mathrm{C}^{18} \mathrm{O}(J=1-0)$ dataset.

The brightest portion of GMC G23.0-0.4, which appears roughly parallel to the Galactic plane, generally displays a long filamentary structure. The dense gas of the GMC traced by $\mathrm{C}^{18} \mathrm{O}(J=1-0)$ emission is primarily distributed in a rectangle region centered at $(l, b$, $v)=\left(23.0,-0.4,77 \mathrm{~km} \mathrm{~s}^{-1}\right)$ (see the red rectangle in Figure 2). Actually, the main axis of GMC G23.0-0.4 is inclined by $20^{\circ}$ with respect to the Galactic plane. The dense filament of GMC G23.0-0.4 extends $~ 1.1$ in length and is distinguished by bright $\mathrm{CO}$ emissions. The mean width of the filamentary GMC traced by $\mathrm{C}^{18} \mathrm{O}(J=1-0)$ emission is about $6^{\prime}$, whereas the width of the thin filament in the denser region is near $2^{\prime}$ or less. It indicates that there are some substructures (e.g., slim filaments, dense cores and clumps) within the filamentary GMC G23.0-0.4 (Figure 2). Future observations at higher spatial resolution will be helpful in investigating these interesting characteristics of the GMC.

In general, the filamentary structure of GMC G23.0-0.4 is hierarchical. In our spatial resolution ( 0'8), GMC G23.0-0.4 displays a wave-like and branch structures along the trunk of the filament. Some thin filaments with $\sim 2^{\prime}$ width and pc-scale dense clumps are distributed along the $77 \mathrm{~km} \mathrm{~s}^{-1}$ giant filament (e.g., see Figures 2, 3, and 4). 


\subsubsection{Physical Parameters of the GMC}

Physical parameters of the GMC can be derived from the following description (e.g., see the appendix in Bourke et al. 1997). Firstly, the excitation temperature of the CO gas $T_{\text {ex }}$ can be derived using the equation:

$$
T_{\mathrm{mb}}=f\left[J\left(T_{\mathrm{ex}}\right)-J\left(T_{\mathrm{bg}}\right)\right][1-\exp (-\tau)]
$$

where $\tau$ is the optical depth, $T_{\mathrm{ex}}$ and $T_{\mathrm{bg}}$ is the excitation temperature and the background temperature, respectively, and

$$
J(T)=\frac{h \nu}{k} \frac{1}{\exp (h \nu / k T)-1} .
$$

Assuming that the ${ }^{12} \mathrm{CO}(J=1-0)$ emission is optically thick $\left(\tau_{12} \gg 1\right)$ and the beam filling factor $f$ of it is equal to 1 , we can get the excitation temperature from the peak temperature of ${ }^{12} \mathrm{CO}(J=1-0)$ emission. The distribution of the excitation temperature is shown in Figure 3. The self-absorption from the cold foreground gas along the LOS is ignored and the derived excitation temperature as a result should be regarded as lower limit.

The optical depth of ${ }^{13} \mathrm{CO}(J=1-0)$ and $\mathrm{C}^{18} \mathrm{O}(J=1-0)$ of GMC G23.0-0.4 can be estimated by assuming that the excitation temperature of these molecular lines have the same value as that of ${ }^{12} \mathrm{CO}$ pixel by pixel (see Figure 3). Thus, in the local thermodynamic equilibrium (LTE) assumption, the optical depths of $\tau_{13}$ and $\tau_{18}$, the column densities of ${ }^{13} \mathrm{CO}$ and $\mathrm{C}^{18} \mathrm{O}$ can be derived using the following equations (e.g., see the appendix in Bourke et al. 1997):

$$
\begin{aligned}
& \tau_{13}(V)=-\ln \left[1-\frac{T_{\mathrm{MB} 13}(V)}{5.29\left[J_{13}\left(T_{\mathrm{ex}}\right)-0.164\right]}\right] \\
& \tau_{18}(V)=-\ln \left[1-\frac{T_{\mathrm{MB} 18}(V)}{5.27\left[J_{18}\left(T_{\mathrm{ex}}\right)-0.167\right]}\right] \\
& N\left({ }^{13} \mathrm{CO}\right)=2.42 \times 10^{14} \sum_{V}\left[\frac{0.2\left(\mathrm{~km} \mathrm{~s}^{-1}\right) \tau_{13}(V) T_{\mathrm{ex}}}{1-\exp \left[-5.29 / T_{\mathrm{ex}}\right]}\right] \mathrm{cm}^{-2},
\end{aligned}
$$

and 


$$
N\left(\mathrm{C}^{18} \mathrm{O}\right)=2.42 \times 10^{14} \sum_{V}\left[\frac{0.2\left(\mathrm{~km} \mathrm{~s}^{-1}\right) \tau_{18}(V) T_{\mathrm{ex}}}{1-\exp \left[-5.27 / T_{\mathrm{ex}}\right]}\right] \mathrm{cm}^{-2}
$$

where $T_{\text {ex }}$ is the excitation temperature of these molecules in $\mathrm{K}$, and $J_{13}\left(T_{\mathrm{ex}}\right)=1 /\left[\exp \left(5.29 / T_{\mathrm{ex}}\right)-\right.$ 1] for ${ }^{13} \mathrm{CO}(J=1-0)$ and $J_{18}\left(T_{\text {ex }}\right)=1 /\left[\exp \left(5.27 / T_{\text {ex }}\right)-1\right]$ for $\mathrm{C}^{18} \mathrm{O}(J=1-0)$. In the calculation, we divide each spectrum into $0.2 \mathrm{~km} \mathrm{~s}^{-1}$ bins to estimate the optical depth in each bin. Accordingly, we can calculate the ${ }^{13} \mathrm{CO}$ and $\mathrm{C}^{18} \mathrm{O}$ column density within the LSR velocity range from 72 to $81 \mathrm{~km} \mathrm{~s}^{-1}$.

The $\mathrm{H}_{2}$ column density of the GMC can be estimated from the optically thin ${ }^{13} \mathrm{CO}$ $(J=1-0)$ and $\mathrm{C}^{18} \mathrm{O}(J=1-0)$ emission. In the above calculation, the ${ }^{13} \mathrm{CO}$ abundance of $N\left(\mathrm{H}_{2}\right) / N\left({ }^{13} \mathrm{CO}\right) \approx 7 \times 10^{5}($ Frerking et al. 1982$)$ and the $\mathrm{C}^{18} \mathrm{O}$ abundance of $N\left(\mathrm{H}_{2}\right) / N\left(\mathrm{C}^{18} \mathrm{O}\right)$ $\approx 7 \times 10^{6}$ (Castets \& Langer 1995) are adopted. On the other hand, using the optically thick ${ }^{12} \mathrm{CO}(J=1-0)$ emission as the tracer of molecular gas, the $\mathrm{H}_{2}$ column density of the GMC can be estimated directly by adopting the mean CO-to- $\mathrm{H}_{2}$ mass conversion factor $1.8 \times 10^{20} \mathrm{~cm}^{-2} \mathrm{~K}^{-1} \mathrm{~km}^{-1} \mathrm{~s}$ (Dame et al. 2001). In the estimation of the mass of the GMC, a mean molecular weight per $\mathrm{H}_{2}$ molecule of 2.76 has been adopted. The distance of the GMCs was adopted as $4.4 \mathrm{kpc}$ (see Section 4.1). The parameters of the filamentary GMC G23.0-0.4 are listed in Table 2.

According to Figure 3, we find that the excitation temperature in the region of the dense filamentary GMC G23.0-0.4 is higher compared to that of the other molecular gas in the FOV. The mean excitation temperature of the filamentary structure is about $20 \mathrm{~K}$ and the excitation temperatures in some interface regions between SNR W41 and SNR G22.7-0.2 are higher than $26 \mathrm{~K}$. The value of the excitation temperature of GMC G23.0-0.4 is comparable to those of other GMCs, e.g., 10-70 K for the Orion-A GMC (Nagahama et al. 1998) and 7$56 \mathrm{~K}$ for the W51 GMC (Parsons et al. 2012). We also note that the excitation temperature of GMC G23.0-0.4 is very close to that in the high density layer of the W3 GMC (15-30 K, Polychroni et al. 2012).

The structures of GMC G23.0-0.4 can be distinguished readily in the maps of the optical depth of the $\mathrm{C}^{18} \mathrm{O}(J=1-0)$ emission (the right panel in Figure 4). We find that the distribution of $\tau_{13}$ is more diffuse and extended than that of $\tau_{18} . \tau_{13}$ in the dense part of GMC G23.0-0.4 is larger than 0.5, which indicates that the optical depth of ${ }^{13} \mathrm{CO}(J=1-$ 0 ) is not optically thin. On the other hand, $\tau_{18}$ is mostly less 0.15 in the region of the filamentary GMC G23.0-0.4. The highest $\tau_{18}$ are $0.21-0.23$ in the high $\tau_{13}$ region, which show the densest parts in the GMC. The distribution of the $\mathrm{C}^{18} \mathrm{O}$ gas is more compact than those of the ${ }^{12} \mathrm{CO}$ and ${ }^{13} \mathrm{CO}$ gas, which indicates that the $\mathrm{C}^{18} \mathrm{O}$ emission can clearly reveal dense structures of GMC G23.0-0.4 indeed. 
The column density, the mass, and the density of the main body of GMC G23.0-0.4 is $1.7-2.9 \times 10^{22} \mathrm{~cm}^{-2}, 3.2-5.2 \times 10^{5} M_{\odot}$, and $730-1200 \mathrm{~cm}^{-3}$, respectively. We note that the column density only represents the mean value for the filamentary GMC in the rectangle region. The column density is higher than $5 \times 10^{22} \mathrm{~cm}^{-2}$ in some dense regions, which indicates that the volume density of these regions is higher than $6 \times 10^{3} \mathrm{~cm}^{-3}$ (assuming a depth of $2^{\prime}$, the mean width of slim filaments, for the dense region). Therefore, the strong $\mathrm{C}^{18} \mathrm{O}(J=1-0)$ emission, which represents the distribution of the high-density molecular gas in the GMC G23.0-0.4, is very likely tracing the potential star formation regions (see Section 4.3.1).

The mass of the GMC listed in Table 2 is probably the lower limit because some diffuse molecular gas outside the rectangle region is not accounted for. Based on the optically thick ${ }^{12} \mathrm{CO}(J=1-0)$ emission, we estimate that the total mass of GMC G23.0-0.4 is $\sim 1.2 \times 10^{6} M_{\odot}$ due to a large amount of low-density molecular gas in the enveloping layer of the GMC, which is outside the rectangle region. In the above calculation, all pixels above $40 \mathrm{~K} \mathrm{~km} \mathrm{~s}^{-1}$ (about the half of the ${ }^{12} \mathrm{CO}$ mean intensity of the GMC) are accounted for and the total mass of the GMC is derived from a $\sim 2200 \operatorname{arcmin}^{2}$ region, in which region the velocity interval is $68-82 \mathrm{~km} \mathrm{~s}^{-1}$ and the mean intensity is $87 \mathrm{~K} \mathrm{~km} \mathrm{~s}^{-1}$, respectively. On the contrary, the dense gas (e.g., $\gtrsim 10^{3}-10^{4} \mathrm{~cm}^{-3}$ ), which only represents a fraction of the GMC's total mass, is mainly located in the trunk of the filamentary GMC G23.0-0.4 (388 $\operatorname{arcmin}^{2}$ in Table 2, also see the right panel of Figure 4).

The filamentary GMC G23.0-0.4 is different from the giant molecular filaments studied by Ragan et al. (2014), in which samples these giant filaments have low dense gas mass fractions $(2 \%-12 \%)$. In the analysis of Ragan et al. (2014), they selected the ${ }^{13} \mathrm{CO}(J=1-0)$ emission $\left(<10^{3} \mathrm{~cm}^{-3}\right)$ as the total masses tracer and the ATLASGAL $870 \mu \mathrm{m}$ dust emission as the dense gas mass tracer $\left(>10^{22} \mathrm{~cm}^{-2}\right)$. In our study, we use ${ }^{12} \mathrm{CO}(J=1-0)$ emission $\left(\sim 10^{2} \mathrm{~cm}^{-3}\right)$ and $\mathrm{C}^{18} \mathrm{O}(J=1-0)$ emission $\left(>10^{22} \mathrm{~cm}^{-2}\right.$, see Figure 9$)$ to calculate the total mass and the dense gas mass of the GMC, respectively. The total mass of the GMC in our study is obviously larger than that from the ${ }^{13} \mathrm{CO}(J=1-0)$ emission, while the mass of the dense gas from the $\mathrm{C}^{18} \mathrm{O}(J=1-0)$ emission is roughly compared to that from the ATLASGAL $870 \mu \mathrm{m}$ dust emission (see the discussion of FP1 in Section 4.3.1). Therefore, GMC G23.0-0.4 seems to be denser than the GMC samples studied by Ragan et al. (2014).

\subsubsection{Velocity Structures of the Dense Molecular Gas}

We made the channel maps of $\mathrm{C}^{18} \mathrm{O}(J=1-0)$ emission in the interval of $69-85 \mathrm{~km} \mathrm{~s}^{-1}$ by step of $2 \mathrm{~km} \mathrm{~s}^{-1}$ to investigate the spatial distribution of the dense molecular gas (Figure 5). The main body of filamentary GMC G23.0-0.4 is in the velocity interval of $75-79 \mathrm{~km} \mathrm{~s}^{-1}$. 
The molecular gas in $71-75 \mathrm{~km} \mathrm{~s}^{-1}$ is assembled in the interface between SNR W41 and SNR G22.7-0.2. On the other hand, the molecular gas in $79-85 \mathrm{~km} \mathrm{~s}^{-1}$ are roughly in the centre of SNR W41.

In the position-velocity (PV) diagrams along the long filamentary GMC G23.0-0.4 (Figure 6), we can discern that the GMC is in the velocity interval of $70-84 \mathrm{~km} \mathrm{~s}^{-1}$. The interaction between SNR G22.7-0.2 and the GMC can be discerned from the ${ }^{12} \mathrm{CO}(J=1-0)$ $\mathrm{PV}$ diagram (see detail in $\mathrm{Su}$ et al. 2014). The $V_{\mathrm{LSR}} \sim 100 \mathrm{~km} \mathrm{~s}^{-1} \mathrm{MC}$ component is from the molecular gas near the tangent point (MC G23.4, Ohishi et al. 2012), which is irrelevant to GMC G23.0-0.4. We also made the PV diagram of the $\mathrm{C}^{18} \mathrm{O}(J=1-0)$ emission along the interface between SNR W41 and SNR G22.7-0.2 (Figure 7). The PV diagram delineates some diffuse emission extended to $70 \mathrm{~km} \mathrm{~s}^{-1}$ besides the dense 74 and $78 \mathrm{~km} \mathrm{~s}^{-1} \mathrm{MCs}$. The feature probably relates to the star forming activity near the region. Based on the spatial distribution of the molecular gas, it seems reasonable to assume that the emission within the velocity of $70-84 \mathrm{~km} \mathrm{~s}^{-1}$ comes from GMC G23.0-0.4 that is associated with the ambient

SNRs ( $\mathrm{Su}$ et al. 2014). The distance estimation of the MCs suggests that the GMC complex is probably in the near side of the Scutum-Centaurus arm (see Section 4.1).

\subsection{Other Interesting MCs}

Besides GMCs G23.0-0.4 (this paper) and G22.6-0.2 (Su et al. 2014), there are some interesting MCs with similar LSR velocity in the FOV. A partial shell structure $\left(l=23^{\circ} .4\right.$, $b=-0.6)$ is ambient to the southern edge of SNR W41, and a series of pillar-like protrusions are along a bright-rim structure near $(l=23.25, b=0.08)$ (Figure 2 ).

The low-density $\left(\sim 10^{2}-10^{3} \mathrm{~cm}^{-3}\right)$ molecular gas of the partial shell structure is in the southern boundary of SNR W41, in which place the remnant shows very faint radio emission (see the left panel in Figure 4). An early-type massive star would create a wind bubble of radius of $56 n_{\mathrm{ISM}}^{-0.3}$ pc (McKee et al. 1984). A radius of such a bubble will be about $23 \mathrm{pc}$, assuming the mean density of the region is $20 \mathrm{~cm}^{-3}$. This value is less than that of the partial shell structure (radius of $\sim 28^{\prime}$ or $36 \mathrm{pc}$ at a distance of $4.4 \mathrm{kpc}$ ). Therefore, the partial shell structure, with a total mass of $\sim 2 \times 10^{5} M_{\odot}$, is likely the result of a series of massive stellar winds. On the other hand, supernova explosions are also probably held responsible for the formation of such structure.

In the northern region of the FOV, some small pillar-like protrusions seem to be located along a partial shell near $\left(l=23^{\circ} 25, b=00^{\circ} 08\right)$. These small pillar-like protrusions have bright ${ }^{13} \mathrm{CO}(J=1-0)$ and $\mathrm{C}^{18} \mathrm{O}(J=1-0)$ emission (see Figure 4$)$, which can be distinguished 
from the diffuse ${ }^{12} \mathrm{CO}(J=1-0)$ surroundings (Figure 2). At the apexes of the pillar-like molecular clouds, bright $870 \mu \mathrm{m}$ dust emission (5.94 Jy for G23.1989+0.0009 and 4.85 Jy for G23.2646+0.0774) are coincident well with the peak emission of $\mathrm{C}^{18} \mathrm{O}(J=1-0)$. The interesting structures are probably related to the energetic radiation of the nearby early-type stars. Such bright-rim clouds are often associated with the photoevaporation from the nearby massive star (e.g., Tavlor et al. 1999; Bally et al. 2010). A diffuse H II region G23.162+0.023 (Lockman et al. 1996), which is indeed located at the west of these pillar-like MCs, is probably responsible for these bright-rim clouds.

Both of the interesting MCs adjacent to GMC G23.0-0.4 are very likely associated with stellar feedback from massive stars. It shows that GMC 23.0-0.4 is sited near the region of massive stars in the view of the adjacent interesting MCs, which is consistent with the result of Section 4.2.

\section{DISCUSSION}

\subsection{Distance of GMC G23.0-0.4}

We use two methods to determine the distance of the dense filamentary GMC G23.0-0.4. In the first method, the distance of the GMC can be derived from the trigonometric parallax assuming the association between the GMC and the $6.7 \mathrm{GHz}$ methanol maser G23.01-0.41. The distance of it is $4.59_{-0.33}^{+0.38} \mathrm{kpc}$ (Brunthaler et al. 2009). It appears reasonable because the $6.7 \mathrm{GHz}$ methanol maser G23.01-0.41, which is just located in the centre of the GMC, has a similar LSR velocity $\left(\mathrm{V}_{\text {maser }} \sim 81 \mathrm{~km} \mathrm{~s}^{-1}\right.$, see Table 2 in Brunthaler et al. 2009) to GMC G23.0-0.4.

In the second method, the distance of the GMC can be estimated from the LSR velocity $\left(\sim 77 \mathrm{~km} \mathrm{~s}^{-1}\right)$ of it. Using the Galactic rotation curve of Reid et al. (2014), we thus place the GMC G23.0-0.4 at a near kinematic distance of $4.4 \pm 0.4 \mathrm{kpc}$. The kinematic distance ambiguity can be resolved from the H I self-absorption method (HISA, see Figures 1 and 2 in Roman-Duval et al. 2009). Based on the HISA method, a molecular cloud located at the near kinematic distance will exhibit the $21 \mathrm{~cm}$ absorption feature that is coincident with the ${ }^{13} \mathrm{CO}$ peak from the cloud (Roman-Duval et al. 2009). These authors suggested that the $\sim 70-82 \mathrm{~km} \mathrm{~s}^{-1}$ MCs (see Table 1 in their paper), which is mostly associated with GMC G23.0-0.4 in the FOV, is located at the near kinematic distance. We also checked the method for other small regions in GMC G23.0-0.4 using VGPS H I data and our CO data and find that the GMC is indeed in the near side. Accordingly, we exclude the far kinematic distance of the GMC, which result is consistent with that of Ellsworth-Bowers et al. (2013). In their 
work, the $\sim 77 \mathrm{~km} \mathrm{~s}^{-1}$ GMC is also in the near side (see Table 3 in Ellsworth-Bowers et al. 2013).

We note that the near kinematic distance of the GMC is in agreement well with the trigonometric distance and that of other works (Leahy \& Tian 2008; Messineo et al. 2010, 2014). Combining the above analysis, we suggest that the dense filamentary GMC G23.0-0.4, together with the rich massive star formation groups ambient to the giant filament (Section 4.2), is probably located at the near side of the Scutum-Centaurus arm in the first quadrant (e.g., please refer to the spiral arm models of the Milky Way, Taylor \& Cordes 1993; Reid et al. 2014). For simplicity, we adopt the value of $4.4 \mathrm{kpc}$ as the distance of the GMC throughout the paper.

\subsection{Environment of GMC G23.0-0.4}

We have shown that SNRs G22.7-0.2 and W41 are both interacting with the $V_{\mathrm{LSR}} \sim 77 \mathrm{~km} \mathrm{~s}^{-1}$ GMC G23.0-0.4 (Su et al. 2014). The kinematic signature of the interaction between SNR G22.7-0.2 and the GMC can be seen from the PV diagram of the ${ }^{12} \mathrm{CO}(J=1-0)$ emission (the left panel of Figure 6). On the other hand, emission of ${ }^{12} \mathrm{CO}(J=3-2)$ is a good tracer of the warm and dense molecular gas associated with star formation. It is also a good tracer of shocked gas, e.g., outflow activity, SNR-MC interaction, and cloud-cloud collision. Unfortunately, the COHRS version 1 only maps a region of $|b|<0.25$ in the direction (Dempsey et al. 2013), which does not cover the main body of GMC G23.0-0.4. Nevertheless, the available ${ }^{12} \mathrm{CO}(J=3-2)$ data are used to study the molecular gas in the FOV. Actually, some enhanced ${ }^{12} \mathrm{CO}(J=3-2)$ emission in the northern region of SNR W41 is detected in the velocity interval $70-74 \mathrm{~km} \mathrm{~s}^{-1}$ (the blueshift compared to the LSR velocity $77 \mathrm{~km} \mathrm{~s}^{-1}$ of the GMC) when we searched for the proof of the SNR-MC interaction. It is consistent with the result that SNR W41 lies behind GMC G23.0-0.4 and the remnant is interacting with the GMC (Frail et al. 2013). We hope that the data of the COHRS release 2 will provide further insight into the nature of the warm and dense molecular gas of the overall GMC G23.0-0.4.

There are multiple overlapping H II regions in the FOV of SNRs G22.7-0.2 and W41 (e.g., Messineo et al. 2010). Even though the velocity of H II regions often deviates from that of their parent MCs, we suggest that most of them with the systematic velocity of 70-81 $\mathrm{km} \mathrm{s}^{-1}$ probably have similar distance to the $77 \mathrm{~km} \mathrm{~s}^{-1}$ GMC (Su et al. 2014). In the right panel of Figure 8, positions of these regions of twelve $\mathrm{H}$ II regions and two H II/SNR complexes (Lockman 1989; Kuchar \& Clark 1997; Giveon et al. 2005a, b; Helfand et al. 2006; Anderson et al. 2011, 2014) are marked with red and blue circles, respectively. Actually, at 
least 6 H II regions (e.g., see Table 6 in Anderson et al. 2014) in the FOV are most likely located at the near distance.

Moreover, the stellar cluster GLIMPSE9 $\left(l=22^{\circ} .756, b=-0.400\right)$ is also associated with GMC G23.0-0.4 (Messineo et al. 2010; Su et al. 2014). Recently, Messineo et al. (2014) discovered a number of massive stars surrounding the cluster GLIMPSE9 in the south of SNR G22.7-0.2 (REG GLIMPSE9Large in their paper) and some massive stars near the centre of SNR W41 (REG4 in their paper), which early-type stars with K-band extinction from $\sim 1.3-1.9 \mathrm{mag}$ have spectrophotometric distances consistent with that of the GMC. The two massive star groups, which are most likely associated with the GMC G23.0-0.4 (Messineo et al. 2010, 2014), are also marked with the blue boxes in Figure 8.

The existence of two SNRs, several H II regions, and a number of massive stars associated with GMC G23.0-0.4 show the energetic star formation activity in the region. The natal GMCs of these objects are very likely destroyed by the process of the photoevaporation of the HII regions (McKee \& Ostriker 2007). On the other hand, the supernova explosions will remove a significant fraction of the cloud mass if the massive progenitors of them did not run far away from their parent GMCs (Iffrig \& Hennebelle 2015). However, these energetic process have a limited influence on the nearby dense gas of the filamentary GMC G23.0-0.4. For example, SNR G22.7-0.2, which's center is about 18 pc away from the dense filamentary GMC, has only $3.3 \times 10^{4} M_{\odot} \mathrm{km} \mathrm{s}^{-1}$ momentum and $5 \times 10^{48}$ erg kinetic energy injection to GMC G23.0-0.4 (Su et al. 2014). The dense gas within GMC G23.0-0.4 still keep the filamentary structure, which is roughly parallel to the Galactic plane.

The formation of GMCs along the spiral arms is believed to be related to the spiral shocks (e.g., Dobbs et al. 2006; Dobbs \& Bonnell 2007). Assuming that GMC G23.0-0.4 and their ambient GMCs, which are the natal places of the massive progenitors of the SNRs, formed simultaneously in the region of the FOV, the age of GMC G23.0-0.4 is $\gtrsim 10 \mathrm{Myr}$ (the typical lifetimes of B1-O9 stars), in which timescale the natal GMCs of SNRs G22.7-0.2 and W41 and the Hir regions (Su et al. 2014) had been destroyed by the violent massive star formation therein (e.g., Section 3.2.2 in McKee \& Ostriker 2007). But these energetic process outside GMC G23.0-0.4 have a limited influence on the dense gas of the GMC. On the other hand, the strong massive star forming activities are ongoing along the filamentary GMC G23.0-0.4 (see Section 4.3), which will exhaust and dissipate the molecular gas of the GMC in future several Myr. Actually, a part of the dense gas has been destroyed by the H II/SNR complex G022.760-0.485 (FP2 in Figure 9, Section 4.3). In the region of the $\mathrm{H}$ II/SNR complex G022.760-0.485, the $\mathrm{C}^{18} \mathrm{O}(J=1-0)$ emission is very weak (see the region of 06 in Figure 8 of Su et al. 2014), which is probably due to the stellar feedback of massive star therein. Accordingly, the lifetime of the dense filamentary GMC should be about 10- 
$20 \mathrm{Myr}$, which is consistent with the result of 1-3 free-fall time of massive GMCs (Murrav 2011).

\subsection{Fragmentation of the Giant Filament}

\subsubsection{Fragmentation on large scale}

Actually, $\mathrm{C}^{18} \mathrm{O}(J=1-0)$ emission is not a good tracer for very high-density molecular gas ( $\gtrsim 10^{5} \mathrm{~cm}^{-3}$ ) due to it's relatively low critical density. Other high-density tracers such as $\mathrm{HCN}$ or $\mathrm{N}_{2} \mathrm{H}^{+}$are helpful in investigating conditions within high-density regions of the GMC $\left(\sim 10^{4}-10^{6} \mathrm{~cm}^{-3}\right)$. In our study, we use the bright dust emission to reveal the dense, massive structures within GMC G23.0-0.4 accordingly. The strong dust emission is labelled on red contours in the left panel of Figure 8. These dense molecular clouds, together with massive star formation regions, probably represent the molecular gas concentration along the dense filament.

The fragmentation points (FPs) 1-5 along GMC G23.0-0.4 are shown in Figure 9. The properties of these FPs are summarized in Table 3. FP1 contains a millimetric radio source BGPS G022.548-00.525 (Rosolowsky et al. 2010), in which region the emission from the $870 \mu \mathrm{m}$ ATLASGAL data is also bright (G022.5483-0.5225, 3.68 Jy; Csengeri et al. 2014). The mass of the dense molecular gas in the region of $1.5 \times 1.5$ estimated from $\mathrm{C}^{18} \mathrm{O}(J=1-0)$ emission is $\sim 4 \times 10^{3} M_{\odot}$. Assuming that the dust can be characterized by a single temperature of $15 \mathrm{~K}$ in the region (see Figure 3 ), we calculate the isothermal mass of $\gtrsim 6 \times 10^{2} M_{\odot}$ in the region of $0.5 \times 0.5$ based on the dust-continuum data. The high surface density ( $\gtrsim 10^{3} M_{\odot} \mathrm{pc}^{-2}$ ) implies the potential star formation in the region of FP1. FP2 is a H II/SNR complex region (Lockman 1989; Kuchar \& Clark 1997; Helfand et al. 2006; Anderson et al. 2011, 2014), which is associated with the GMC G23.0-0.4 (Messineo et al. 2010, 2014; Su et al. 2014). FP3 and FP4, contains the 6.7 GHz methanol masers G23.01-0.41 and G23.19-0.38, respectively, which are the good tracers of massive star formation (Szvmczak et al. 2002; Brunthaler et al. 2009). In the two regions, the $1.1 \mathrm{~mm}$ (Rosolowsky et al. 2010) and $870 \mu \mathrm{m}$ (Csengeri et al. 2014) emission are very bright (e.g., BGPS G023.012-00.410 and ATLASGAL G023.0063-0.3991 (3.79 Jy), and G023.0082-0.4092 (12.76 Jy) in FP3; BGPS G023.208-00.378 and ATLASGAL G023.2056-0.3772 (11.30 Jy) and G023.2122-0.3726 (1.34 Jy) in FP4). FP5 is similar to that of FP1, in which place a millimetric radio source BGPS G023.368-00.290 is in the region (Rosolowsky et al. 2010). The emission from the $870 \mu \mathrm{m}$ ATLASGAL data is also bright (G023.3652-0.2887, $4.93 \mathrm{Jy}$; Csengeri et al. 2014) and many young stellar objects (YSOs) are distributed in the vicinity of FP5 (Ragan et al. 2009). 
We also showed the abundance ratio distribution between ${ }^{13} \mathrm{CO}$ and $\mathrm{C}^{18} \mathrm{O}, X^{13} \mathrm{CO} / X_{\mathrm{C}^{18} \mathrm{O}}$ for the $77 \mathrm{~km} \mathrm{~s}^{-1}$ molecular gas in the left panel of Figure 8 . We found that the abundance ratio $X_{13 \mathrm{CO}} / X_{\mathrm{C}^{18} \mathrm{O}}$ becomes lower (similar to the value of 5.5 in the Solar system, see Table 4 in Wilson \& Rood 1994) in the regions of the bright dust-continuum emission (the red contours in the left panel of Figure 8), which probably indicates the high-density regions for star formation. The difference between the abundance ratios in the bright dust emission regions (e.g., $\sim 5$ for FPs 1, 3, 4, and 5) and the other region (e.g., $\sim 10$ for FP 2) very likely represents the evolutionary sequence of the dense molecular gas in the GMC. The abundance ratio will be relatively higher when the dense molecular cores are chemically influenced by the far ultraviolet (FUV) radiation from the massive stars embedded in the GMC (van Dishoeck \& Black 1988). In other words, The photodissociation rate of the $\mathrm{C}^{18} \mathrm{O}$ molecule is larger than that for ${ }^{13} \mathrm{CO}$, which leads to the higher abundance ratio $X_{{ }^{13} \mathrm{CO}} / X_{\mathrm{C}^{18} \mathrm{O}}$ in the massive star's UV radiation field. It is consistent with the result that a part of the dense gas in GMC G23.0-0.4 has been destroyed by the H II/SNR complex G022.760-0.485 (see Section 4.2). In contrast, the lower abundance ratio represents the earlier stage of dense MCs, which are not or less affected by massive star formation. The low abundance ratio in the bright dust emission regions is also consistent with the result that most of cold dust emission is indeed from $77 \mathrm{~km} \mathrm{~s}^{-1}$ GMCs (see Figure 8). We find that the distribution of the dust clumps/cores (the fifteen black boxes in Figure 8) is coincident well with that of the $\mathrm{C}^{18} \mathrm{O}$ emission. In the FOV, 11/15 dust clumps are associated with GMC G23.0-0.4. The distance of these dust-continuum-identified MC clumps (Ellsworth-Bowers et al. 2013) is therefore consistent with that of the $77 \mathrm{~km} \mathrm{~s}^{-1}$ MCs.

The mean column density from $\mathrm{C}^{18} \mathrm{O}(J=1-0)$ emission is $0.5-1.5 \times 10^{23} \mathrm{~cm}^{-2}$ for the regions of FP1, FP3, FP4, and FP5, in which regions the column density from $\mathrm{NH}_{3}$ emission are all higher than $1 \times 10^{23} \mathrm{~cm}^{-2}$ (Wienen et al. 2012). The FP1, FP3, FP4, and FP5 indeed show the densest regions along the filament (see the red contours in the left panel of Figure 8), whereas the dense molecular gas in FP2 is exhausted and dissipated by the very strong star formation activities there. The abundance ratio $X_{{ }^{13} \mathrm{CO}} / X_{\mathrm{C}^{18} \mathrm{O}}$ is higher than 10 in the region of FP2, which is consistent with the result that the abundance ratio becomes higher in the mature $\mathrm{H}$ II region within the GMC.

It is worth noting that the column density of $\mathrm{FP} 1$ derived from the $\mathrm{C}^{18} \mathrm{O}$ emission is comparable to that from the $870 \mu \mathrm{m}$ dust emission. According to Kauffmann \& Pillai (2010), both FP1 and FP5 are potentially dense enough $\left(m_{\text {clump }}>m_{\lim }[r]=870 M_{\odot}[r / p c]^{1.33}\right)$ to form massive stars. On the other hand, the $870 \mu \mathrm{m}$ fluxes of FP1 (3.68 Jy) and FP5 (4.93 Jy) are just close to the conservative criterion of $5 \mathrm{Jy}$ at $4.5 \mathrm{kpc}$, which is the flux limit at the distance to potentially form high-mass stars (Csengeri et al. 2014). 
Therefore, two dense clumps potentially sustain high-mass star formation (FP1 and FP5), two ongoing massive stars (FP3 and FP4), and one mature H II/SNR complex region (FP2), which are all the dense gas concentrations associated with the filament, can well represent the fragmentation on large scales $(\sim 10 \mathrm{pc})$ along the dense filamentary GMC G23.0-0.4.

\subsubsection{Turbulence Dominates the Large-scale Fragmentation}

We presented the main physical properties of the dense filament GMC G23.0-0.4 in Section 3.1. The large-scale fragmentation of the dense gas along the filament was discussed in Section 4.3.1. In the section, we investigate the stability of the dense filament.

If we regard the dense gas traced by the $\mathrm{C}^{18} \mathrm{O}(J=1-0)$ emission as a whole, the stability of the filamentary GMC can be described by the virial parameter $\alpha=M_{\mathrm{vir}} / M=2 \sigma_{v}^{2} l / G M$ (Bertoldi \& McKee 1992; Fiege \& Pudritz 2000), where $\sigma_{v}=\Delta V_{\mathrm{C} 18 \mathrm{O}} / 2.355, l$, and $G$ are the average velocity dispersion of $\mathrm{C}^{18} \mathrm{O}(J=1-0)$, the length of the dense filament, and the gravitational constant, respectively. The virial parameter $\alpha$ thus is estimated to be 0.27 , which indicates that the dense filament is gravitationally bound. Then the dense GMC is unstable and will collapse on a free-fall timescale if no other supporting mechanisms are included in the whole system. In the above calculation, we take the long dense GMC as a symmetrica cylinder. The parameters of the cylinder $\left(\sim 66^{\prime} \times 6^{\prime} \times 6^{\prime}\right)$ can be obtained from the CO observations (see Section 3.1.1 and Table 2). The $\sim 66^{\prime}$ length of GMC G23.0-0.4 can be gotten from the ${ }^{13} \mathrm{CO}$ emission $\left(\sim 1.2 \mathrm{~K}\right.$ cutoff of ${ }^{13} \mathrm{CO}$ emission, see the second contour level in the middle panel of Figure 6). Assuming the length of the $\mathrm{C}^{18} \mathrm{O}$ emission of $\sim 66^{\prime}$, the mean width of the dense filament is Area $\left(\mathrm{C}^{18} \mathrm{O}\right) /$ length $\sim 6^{\prime}$. The morphology of the dense filament also can be seen in Figures 4 and 8, which clearly show the boundary of the GMC. We also note that the virial parameter of the GMC from the ${ }^{13} \mathrm{CO}$ emission is similar to that from the $\mathrm{C}^{18} \mathrm{O}$ emission. It is normal since the value of the length, the velocity dispersion, and the total mass of the GMC from ${ }^{13} \mathrm{CO}$ and $\mathrm{C}^{18} \mathrm{O}$ are similar (see Table 2).

Moreover, we can estimate the fragmentational separation within the large-scale filamentary structure to determine the character of the gaseous cylinder. Adopting $10^{3} \mathrm{~cm}^{-3}$ as the mean density of the GMC (Table 2), the filament scale height $H=\sigma_{v}\left(4 G \pi \rho_{c}\right)^{-0.5}$ is about $0.8 \mathrm{pc}$ due to the sausage instability of a self-gravitating fluid cylinder (Chandrasekhar \& Fermi 1953; Inutsuka \& Mivama 1992). In the above calculation, we use the mean full width at half-maximum (FWHM) of $\mathrm{C}^{18} \mathrm{O}(J=1-0)$ emission $\left(\Delta V_{\mathrm{C} 18 \mathrm{O}}=3.5 \mathrm{~km} \mathrm{~s}^{-1}\right)$ in the GMC to

estimate the velocity dispersion of the turbulence (Fiege \& Pudritz 2000). It leads to a 
spacing of $22 H \sim 17 \mathrm{pc}(\sim 0.22$ at $4.4 \mathrm{kpc})$ between the fragmentation clumps, which result is in good agreement with the observations of the fragmentation spacing $0.18-0.26$ along the dense filamentary GMC G23.0-0.4 (see Figure 9). It indicates that the non-thermal turbulent pressure dominates over thermal pressure, which is consistent with other works (e.g., Jackson et al. 2010; Kainulainen et al. 2013). In such case, the turbulence seems to control the fragmentation of the dense gaseous filament on large scale.

The regions of the five FPs represent the nodes of the filamentary GMC, in which regions a large amount of molecular gas fragments into an assembly of cores of subsequent protostars (see the sketch of Figure 2 in Jackson et al. 2010). If these cores are massive and dense enough to form high-mass stars, the stellar feedback such as outflows, UV radiation field, stellar winds, and supernova explosions within the dense filament will dramatically affect the surrounding molecular gas. FPs 2-4 in GMC G23.0-0.4 is probably in such case. The mass in these regions is indeed massive enough to form massive stars. FP2, with its mature H II/SNR complex region, represents a slightly older generation than FP3 and FP4. The dense molecular gas in the region of FP2 was now exhausted, whereas the dense molecular gas in regions of FP3 and FP4 is still plentiful (Figures 8 and 9). Actually, recent massive star formation are ongoing in FP3 and FP4, which are revealed by the $6.7 \mathrm{GHz}$ methanol masers (Szymczak et al. 2002). FPs 1 and 5, which are at both ends of the dense filament, are also massive and dense enough to form massive stars (Section 4.3.1).

\section{SUMMARY}

We have presented a detail CO line study of the dense filamentary GMC G23.0-0.4. Combining the molecular line observations and the $870 \mu \mathrm{m}$ dust continuum emission, the main results are summarized as follows:

1. GMC G23.0-0.4 with LSR velocity of $77 \mathrm{~km} \mathrm{~s}^{-1}$ displays filamentary structure roughly along the Galactic plane, which also shows hierarchical branch structure in $\mathrm{C}^{18} \mathrm{O}(J=1-$ $0)$ emission. Some slim filaments and pc-scale dense clumps are distributed along the main body of the GMC.

2, The optical depth of ${ }^{13} \mathrm{CO}(J=1-0), \tau_{13}$, is higher than 0.5 in the dense parts of the giant molecular filament, while $\tau_{18}$ is only close to 0.2 in the same regions. It indicates that $\mathrm{C}^{18} \mathrm{O}$ is indeed optically thin in the dense regions of the GMC. The dense parts of the GMC is readily seen in $\mathrm{C}^{18} \mathrm{O}(J=1-0)$ emission, which is also the good tracer of the high-density molecular gas $\left(\gtrsim 5 \times 10^{22} \mathrm{~cm}^{-3}\right.$ in our case, see Figure 9$)$.

3, The mass of the dense part of GMC G23.0-0.4 is $\sim 5 \times 10^{5} M_{\odot}$ in the region of 
$\sim 84 \times 15 \mathrm{pc}$, while the total mass of the GMC is probably close to $1.2 \times 10^{6} \mathrm{M}_{\odot}$.

4, GMC G23.0-0.4, which has a distance of $4.4 \mathrm{kpc}$, is probably located at the near side of the Scutum-Centaurus arm in the inner Galaxy. The long structure at $b \sim-0.4$, which is traced by $\mathrm{C}^{18} \mathrm{O}(J=1-0)$ emission along the longitude, is probably related to the density wave of a spiral arm of the Milky Way.

5, There are at least two SNRs, several H II regions, and many massive stars associated with the $77 \mathrm{~km} \mathrm{~s}^{-1}$ molecular gas, indicating massive star formation occurred several Myr ago in the region. The natal GMCs of these objects probably have been destroyed by the strong stellar feedback. Meanwhile, these energetic sources are interacting with the nearby GMC G23.0-0.4.

6 , The distribution of the abundance ratio $\left(X^{13} \mathrm{CO} / X_{\mathrm{C}^{18} \mathrm{O}}\right)$ is coincident well with the distribution of the cold dust emission along the dense filamentary GMC G23.0-0.4. The giant filament is massive and dense enough to form high-mass stars. Actually, massive star formation is ongoing in the nodes of the giant filament, which is also traced by the bright dustcontinuum emission, the $6.7 \mathrm{GHz}$ methanol maser, and $\mathrm{H}$ II $+\mathrm{H}$ II/SNR complex associated with the dense GMC. The fragmentation with spacing $\sim 0.22$ along the filamentary GMC G23.0-0.4 also can be explained by the sausage instability of the cylinder.

7, The turbulence seems to control the fragmentation process of the dense gaseous filament on large scale. Combing the millimeter and IR dataset, we show that massive star formation is ongoing at the fragmentation points with the periodic spacing along the entire filament. The massive stellar feedback near the dense filament has little influence on the evolution of the filamentary GMC G23.0-0.4. On the contrary, the evolutionary processes of the massive stars within the massive filament dominate the fate of the dense GMC.

The authors acknowledge the staff members of the Qinghai Radio Observing Station at Delingha for their support of the observations. We would like to thank the anonymous referee for the critical comments and suggestions which helped to improve the paper. This work is supported by NSFC grants 11233001 and 11233007 . The work is a part of the MultiLine Galactic Plane Survey in CO and its Isotopic Transitions, also called the Milky Way Imaging Scroll Painting, which is supported by the Strategic Priority Research Program, the Emergence of Cosmological Structures of the Chinese Academy of Sciences, grant No. XDB09000000. 


\section{REFERENCES}

Anderson, L. D., Bania, T. M., Balser, D. S., et al. 2014, ApJS, 212, 1

Anderson, L. D., Bania, T. M., Balser, D. S., \& Rood, R. T. 2011, ApJS, 194, 32

André, P., Könyves, V., Arzoumanian, D., \& Palmeirim, P. 2014, Astrophysics and Space Science Proceedings, 36, 225

Bally, J., Anderson, L. D., Battersby, C., et al. 2010, A\&A, 518, L90

Benjamin, R. A., Churchwell, E., Babler, B. L., et al. 2005, ApJ, 630, L149

Bertoldi, F., \& McKee, C. F. 1992, ApJ, 395, 140

Bourke, T. L., Garay, G., Lehtinen, K. K., et al. 1997, ApJ, 476, 781

Brunthaler, A., Reid, M. J., Menten, K. M., et al. 2009, ApJ, 693, 424

Busquet, G., Zhang, Q., Palau, A., et al. 2013, ApJ, 764, L26

Castets, A., \& Langer, W. D. 1995, A\&A, 294, 835

Caswell, J. L., Vaile, R. A., Ellingsen, S. P., Whiteoak, J. B., \& Norris, R. P. 1995, MNRAS, 272,96

Chandrasekhar, S., \& Fermi, E. 1953, ApJ, 118, 116

Contreras, Y., Schuller, F., Urquhart, J. S., et al. 2013, A\&A, 549, A45

Csengeri, T., Urquhart, J. S., Schuller, F., et al. 2014, A\&A, 565, A75

Dame, T. M., Hartmann, D., \& Thaddeus, P. 2001, ApJ, 547, 792

Dempsey, J. T., Thomas, H. S., \& Currie, M. J. 2013, ApJS, 209, 8

Dobashi, K., Matsumoto, T., Shimoikura, T., et al. 2014, ApJ, 797, 58

Dobbs, C. L., \& Bonnell, I. A. 2007, MNRAS, 376, 1747

Dobbs, C. L., Bonnell, I. A., \& Pringle, J. E. 2006, MNRAS, 371, 1663

Ellsworth-Bowers, T. P., Glenn, J., Rosolowsky, E., et al. 2013, ApJ, 770, 39

Fiege, J. D., \& Pudritz, R. E. 2000, MNRAS, 311, 85

Frail, D. A., Claussen, M. J., \& Méhault, J. 2013, ApJ, 773, L19 
Frerking, M. A., Langer, W. D., \& Wilson, R. W. 1982, ApJ, 262, 590

Giveon, U., Becker, R. H., Helfand, D. J., \& White, R. L. 2005a, AJ, 129, 348

-. 2005b, AJ, 130, 156

Goodman, A. A., Alves, J., Beaumont, C. N., et al. 2014, ApJ, 797, 53

Green, D. A. 2014, Bulletin of the Astronomical Society of India, 42, 47

H. E. S. S. Collaboration, Abramowski, A., Acero, F., et al. 2015a, MNRAS, 446, 1163

H. E. S. S. Collaboration, Abramowski, A., Aharonian, F., et al. 2015b, A\&A, 574, A27

Hammersley, P. L., Garzón, F., Mahoney, T. J., López-Corredoira, M., \& Torres, M. A. P. 2000, MNRAS, 317, L45

Helfand, D. J., Becker, R. H., White, R. L., Fallon, A., \& Tuttle, S. 2006, AJ, 131, 2525

Iffrig, O., \& Hennebelle, P. 2015, A\&A, 576, A95

Inutsuka, S.-I., \& Miyama, S. M. 1992, ApJ, 388, 392

Jackson, J. M., Finn, S. C., Chambers, E. T., Rathborne, J. M., \& Simon, R. 2010, ApJ, 719, L185

Jackson, J. M., Rathborne, J. M., Shah, R. Y., et al. 2006, ApJS, 163, 145

Kainulainen, J., Ragan, S. E., Henning, T., \& Stutz, A. 2013, A\&A, 557, A120

Kauffmann, J., \& Pillai, T. 2010, ApJ, 723, L7

Kuchar, T. A., \& Clark, F. O. 1997, ApJ, 488, 224

Leahy, D. A., \& Tian, W. W. 2008, AJ, 135, 167

Li, G.-X., Wyrowski, F., Menten, K., \& Belloche, A. 2013, A\&A, 559, A34

Lockman, F. J. 1989, ApJS, 71, 469

Lockman, F. J., Pisano, D. J., \& Howard, G. J. 1996, ApJ, 472, 173

McKee, C. F., \& Ostriker, E. C. 2007, ARA\&A, 45, 565

McKee, C. F., van Buren, D., \& Lazareff, B. 1984, ApJ, 278, L115

Messineo, M., Figer, D. F., Davies, B., et al. 2010, ApJ, 708, 1241 
Messineo, M., Menten, K. M., Figer, D. F., et al. 2014, A\&A, 569, A20

Murray, N. 2011, ApJ, 729, 133

Nagahama, T., Mizuno, A., Ogawa, H., \& Fukui, Y. 1998, AJ, 116, 336

Ohishi, Y., Sorai, K., \& Habe, A. 2012, PASJ, 64, 74

Parsons, H., Thompson, M. A., Clark, J. S., \& Chrysostomou, A. 2012, MNRAS, 424, 1658

Polychroni, D., Moore, T. J. T., \& Allsopp, J. 2012, MNRAS, 422, 2992

Ragan, S. E., Bergin, E. A., \& Gutermuth, R. A. 2009, ApJ, 698, 324

Ragan, S. E., Henning, T., Beuther, H., Linz, H., \& Zahorecz, S. 2015, A\&A, 573, A119

Ragan, S. E., Henning, T., Tackenberg, J., et al. 2014, A\&A, 568, A73

Reid, M. J., Menten, K. M., Brunthaler, A., et al. 2014, ApJ, 783, 130

Roman-Duval, J., Jackson, J. M., Heyer, M., et al. 2009, ApJ, 699, 1153

Rosolowsky, E., Dunham, M. K., Ginsburg, A., et al. 2010, ApJS, 188, 123

Schneider, N., Csengeri, T., Hennemann, M., et al. 2012, A\&A, 540, L11

Schuller, F., Menten, K. M., Contreras, Y., et al. 2009, A\&A, 504, 415

Shan, W. L., Yang, J., Shi, S. C., et al. 2012, IEEE Transactions on Terahertz Science and Technology, 2, 593

Solomon, P. M., Sanders, D. B., \& Rivolo, A. R. 1985, ApJ, 292, L19

Stark, A. A., \& Lee, Y. 2006, ApJ, 641, L113

Stil, J. M., Taylor, A. R., Dickey, J. M., et al. 2006, AJ, 132, 1158

Su, Y., Yang, J., Zhou, X., Zhou, P., \& Chen, Y. 2014, ApJ, 796, 122

Szymczak, M., Hrynek, G., \& Kus, A. J. 2000, A\&AS, 143, 269

Szymczak, M., Kus, A. J., Hrynek, G., Kěpa, A., \& Pazderski, E. 2002, A\&A, 392, 277

Takahashi, S., Ho, P. T. P., Teixeira, P. S., Zapata, L. A., \& Su, Y.-N. 2013, ApJ, 763, 57

Taylor, A. R., Irwin, J. A., Matthews, H. E., \& Heyer, M. H. 1999, ApJ, 513, 339 
Taylor, J. H., \& Cordes, J. M. 1993, ApJ, 411, 674

van Dishoeck, E. F., \& Black, J. H. 1988, ApJ, 334, 771

Walch, S. K. 2014, Astrophysics and Space Science Proceedings, 36, 173

Wienen, M., Wyrowski, F., Schuller, F., et al. 2012, A\&A, 544, A146

Wilson, T. L., \& Rood, R. 1994, ARA\&A, 32, 191

Yonekura, Y., Asayama, S., Kimura, K., et al. 2005, ApJ, 634, 476

This preprint was prepared with the AAS LATEX macros v5.2. 
Table 1. Details of the Survey Data Used in the Paper

\begin{tabular}{|c|c|c|c|c|c|}
\hline Name & Survey Range & Line/Continuum & Resolution & $\mathrm{rms}$ & Reference \\
\hline COHRS & $\begin{array}{c}10^{\circ}<l<65^{\circ} \\
-0.25 \leq b \leq 0^{\circ} 25\end{array}$ & ${ }^{12} \mathrm{CO}(J=3-2)$ & $16^{\prime \prime}, 1 \mathrm{~km} \mathrm{~s}^{-1}$ & $\sim 1 \mathrm{~K}$ & 1 \\
\hline GRS & $\begin{array}{l}18^{\circ}<l<55^{\circ} .7 \\
-1^{\circ}<b<1^{\circ}\end{array}$ & ${ }^{13} \mathrm{CO}(J=1-0)$ & $46^{\prime \prime}, 0.2 \mathrm{~km} \mathrm{~s}^{-1}$ & $\sim 0.3 \mathrm{~K}$ & 2 \\
\hline VGPS & $\begin{array}{l}18^{\circ}<l<67^{\circ} \\
-1.2<l<1.2\end{array}$ & $\begin{array}{c}\text { H I line } \\
21 \mathrm{~cm} \text { continuum }\end{array}$ & $\begin{array}{c}1^{\prime}, 1.56 \mathrm{~km} \mathrm{~s}^{-1} \\
1^{\prime}\end{array}$ & $\begin{array}{c}\sim 2 \mathrm{~K} \\
\sim 0.3 \mathrm{~K}\end{array}$ & 3 \\
\hline ATLASGAL & $\begin{aligned}-80^{\circ} & <l<60^{\circ} \\
-1^{\circ} & <b<1^{\circ}\end{aligned}$ & $870 \mu \mathrm{m}$ continuum & $16^{\prime \prime} 7$ & $\sim 50-70 \mathrm{mJy} /$ beam & $4,5,6$ \\
\hline
\end{tabular}

Note. - (1) Dempsev et al. 2013; (2) Jackson et al. 2006; (3) Stil et al. 2006; (4) Schuller et al. 2009; (5) Contreras et al. 2013; (6) Csengeri et al. 2014.

Table 2. Properties of GMC G23.0-0.4

\begin{tabular}{ccccc}
\hline \hline $\begin{array}{c}\text { Molecule } \\
\text { tracer }\end{array}$ & $\begin{array}{c}\text { Area } \\
\left(\operatorname{arcmin}^{2}\right)\end{array}$ & $\begin{array}{c}N\left(\mathrm{H}_{2}\right) \\
\left(10^{22} \mathrm{~cm}^{-2}\right)\end{array}$ & $\begin{array}{c}M\left(\mathrm{H}_{2}\right)^{\mathrm{b}} \\
\left(10^{5} M_{\odot}\right)\end{array}$ & $\begin{array}{c}n\left(\mathrm{H}_{2}\right)^{\mathrm{b}, \mathrm{c}} \\
\left(\mathrm{cm}^{-3}\right)\end{array}$ \\
\hline${ }^{12} \mathrm{CO}(J=1-0)$ & 877 & 2.1 & $5.2 d_{4.4}^{2}$ & $890 d_{4.4}^{-1}$ \\
${ }^{13} \mathrm{CO}(J=1-0)$ & 863 & 1.7 & $4.2 d_{4.4}^{2}$ & $730 d_{4.4}^{-1}$ \\
$\mathrm{C}^{18} \mathrm{O}(J=1-0)$ & 388 & 2.9 & $3.2 d_{4.4}^{2}$ & $1200 d_{4.4}^{-1}$ \\
\hline
\end{tabular}

Note. $-{ }^{a}$ See text for the methods used for the calculation. ${ }^{b}$ Parameter $d_{4.4}$ is the distance to the GMC in units of $4.4 \mathrm{kpc}$. ${ }^{c}$ Assuming a depth of $6^{\prime}$, the mean width of the filament in $\mathrm{C}^{18} \mathrm{O}(J=1-0)$ emission, for GMC G23.0-0.4 along the LOS.

Table 3. Characters of the fragmentation nodes along GMC G23.0-0.4

\begin{tabular}{|c|c|c|c|c|c|}
\hline Name & $(l, b)$ & Tracer ${ }^{\mathrm{a}}$ & $T_{\mathrm{ex}} \mathrm{b}, \mathrm{c}$ & $\frac{X_{13} \mathrm{CO}}{X_{\mathrm{C} 18 \mathrm{O}}} \mathrm{c}$ & Evolutionary stage \\
\hline FP1 & $(22.55,-0.52)$ & $\begin{array}{l}\text { bright dust-continuum emission }(3.76 \mathrm{Jy} \text { at } 870 \mu \mathrm{m})^{1,2} \\
\text { high mass clumps from } \mathrm{NH}_{3}\left(>1 \times 10^{23} \mathrm{~cm}^{-2}\right)^{3}\end{array}$ & $\sim 15 \mathrm{~K}$ & $\sim 4-5$ & early stage \\
\hline FP2 & $(22.76,-0.48)$ & H II/SNR complex ${ }^{4,5,6,7}$ & $\sim 20 \mathrm{~K}$ & $\sim 10$ & mature stage \\
\hline FP3 & $(23.01,-0.41)$ & $\begin{array}{c}\text { bright dust-continuum emission }(12.76 \mathrm{Jy} \text { at } 870 \mu \mathrm{m})^{1,2} \\
\text { high mass clumps from } \mathrm{NH}_{3}\left(>3 \times 10^{23} \mathrm{~cm}^{-2}\right)^{3} \\
6.7 \mathrm{GHz} \text { methanol maser } 8,9,10\end{array}$ & $\sim 25 \mathrm{~K}$ & $\sim 4$ & $\begin{array}{l}\text { ongoing massive } \\
\text { star formation }\end{array}$ \\
\hline FP4 & $(23.20,-0.38)$ & $\begin{array}{c}\text { bright dust-continuum emission }(11.3 \mathrm{Jy} \text { at } 870 \mu \mathrm{m})^{1,2} \\
\text { high mass clumps from } \mathrm{NH}_{3}\left(>3 \times 10^{23} \mathrm{~cm}^{-2}\right)^{3} \\
6.7 \mathrm{GHz} \text { methanol maser } 9,11\end{array}$ & $\sim 20 \mathrm{~K}$ & $\sim 4-5$ & $\begin{array}{l}\text { ongoing massive } \\
\text { star formation }\end{array}$ \\
\hline FP5 & $(23.36,-0.29)$ & $\begin{array}{l}\text { bright dust-continuum emission }(4.93 \mathrm{Jy} \text { at } 870 \mu \mathrm{m})^{1,2} \\
\text { high mass clumps from } \mathrm{NH}_{3}\left(>1 \times 10^{23} \mathrm{~cm}^{-2}\right)^{3}\end{array}$ & $\sim 15 \mathrm{~K}$ & $\sim 5-7$ & early stage \\
\hline
\end{tabular}

Note. $\quad{ }^{a}$ (1) Rosolowsky et al. 2010; (2) Csengeri et al. 2014; (3) Wienen et al. 2012; (4) Kuchar \& Clark 1997; (5) Helfand et al. 2006; (6) Anderson et al. 2011; (7) Anderson et al. 2014; (8) Caswell et al. 1995; (9) Szymczak et al. 2002; (10) Brunthaler et al. 2009; (11) Szymczak et al. 2000. ${ }^{b}$ Estimated from the peak temperature of ${ }^{12} \mathrm{CO}(J=1-0) .{ }^{c}$ This work. 


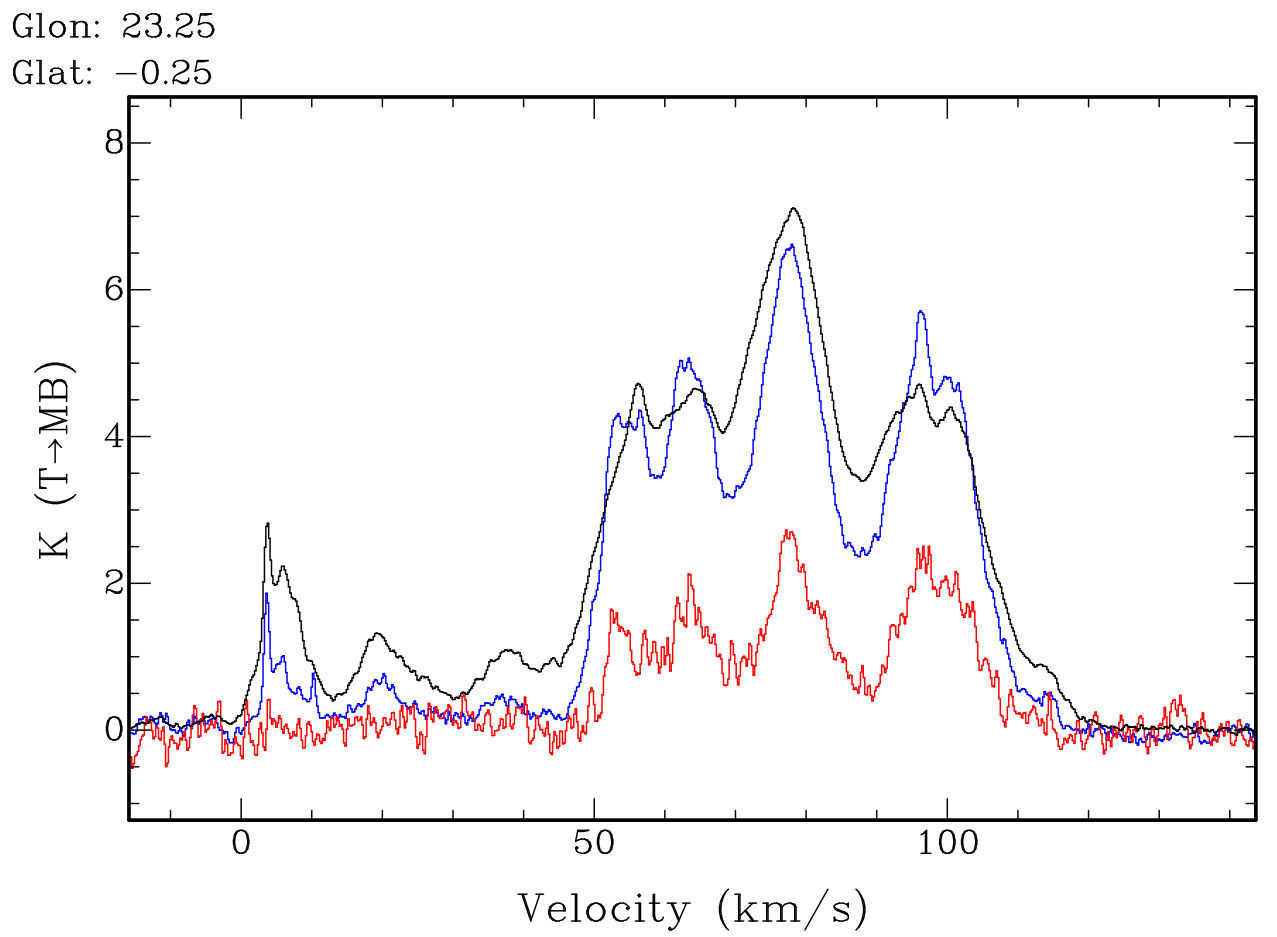

Fig. 1. $-{ }^{12} \mathrm{CO}(J=1-0$; black $),{ }^{13} \mathrm{CO}(J=1-0$; blue, multiplied by a factor of four $)$, and $\mathrm{C}^{18} \mathrm{O}(J=1-0$; red, multiplied by a factor of ten $)$ spectra of the $30^{\prime} \times 30^{\prime}$ region. 


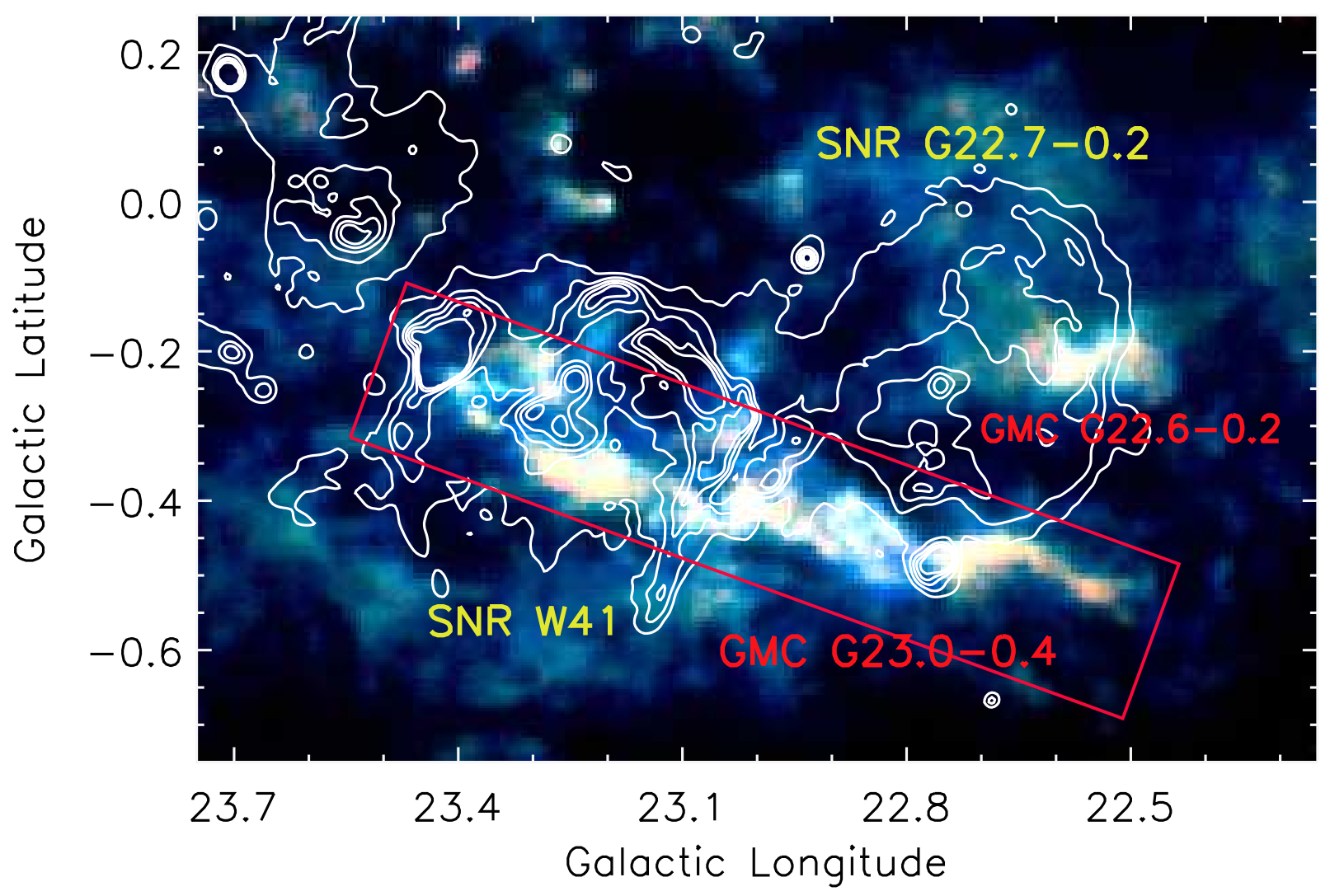

Fig. 2.- ${ }^{12} \mathrm{CO}(J=1-0$; blue $),{ }^{13} \mathrm{CO}(J=1-0$; green $)$, and $\mathrm{C}^{18} \mathrm{O}(J=1-0$; red $)$ intensity maps in the $74-79 \mathrm{~km} \mathrm{~s}^{-1}$ interval with a linear scale in the 1.5 square degree region overlaid with the VGPS $1.4 \mathrm{GHz}$ radio continuum emission contours. The red rectangle shows the region of GMC G23.0-0.4. SNR W41 (G23.3-0.3) and SNR G22.7-0.2 associated with the GMC (Su et al. 2014) are labeled. 


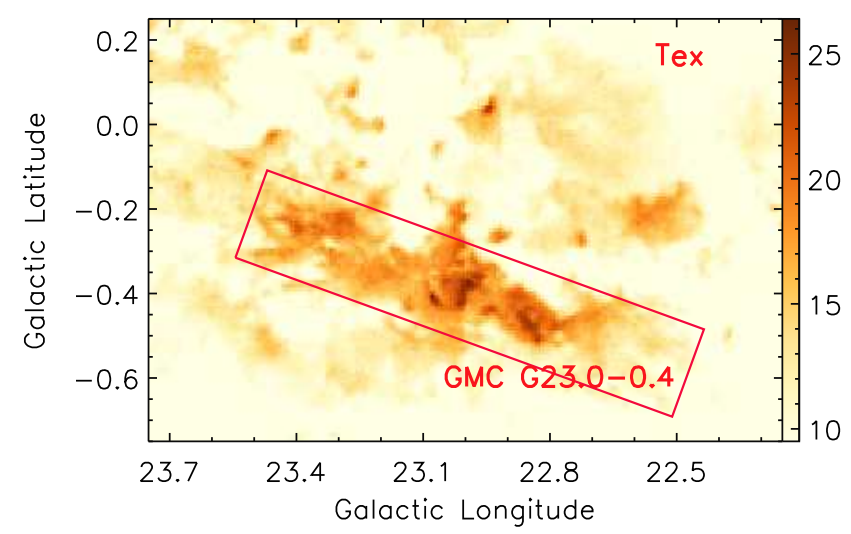

Fig. 3.- Map of the excitation temperature with a linear scale in units of $\mathrm{K}$ from the optically thick ${ }^{12} \mathrm{CO}(J=1-0)$ emission in the $74-79 \mathrm{~km} \mathrm{~s}^{-1}$ interval. The red rectangle shows the region of GMC G23.0-0.4.
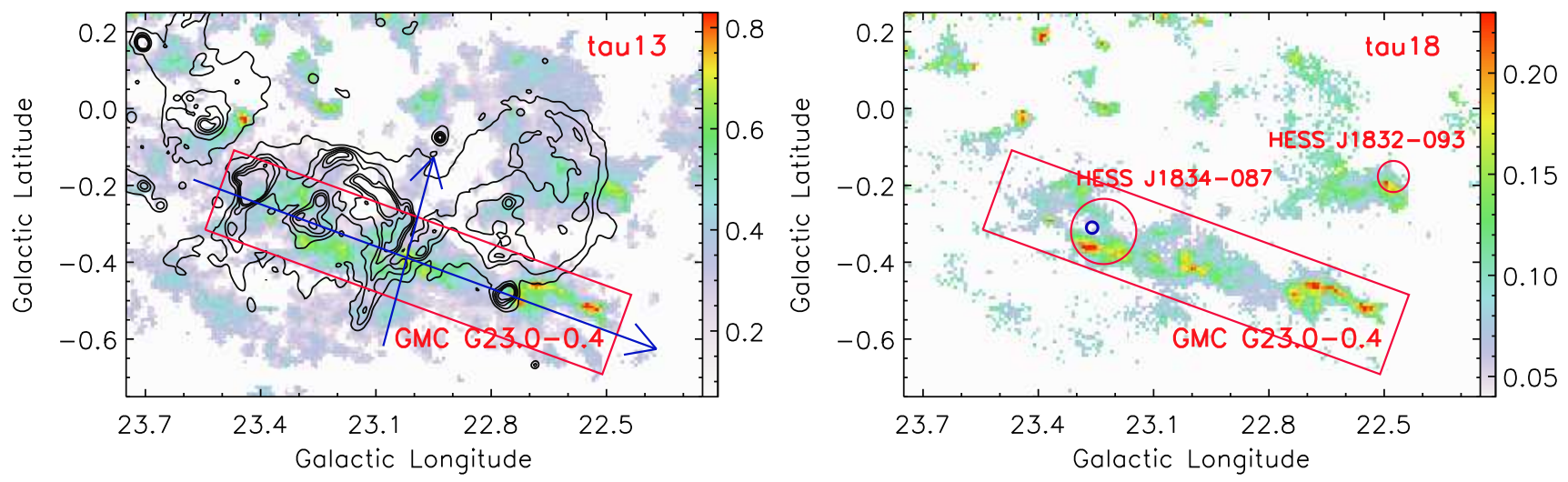

Fig. 4. - Maps of the optical depths of the ${ }^{13} \mathrm{CO}(J=1-0)$ (left, with linear scale) and $\mathrm{C}^{18} \mathrm{O}(J=1-0)$ (right, with square root scale). Note that the intensity scale of right image is different from the left one to highlight the GMC structures. The blue arrows in left panel show the direction of the PV diagrams (see Figures 6,7). The red circles in the right panel show the locations of HESS J1834-087 (H. E. S. S. Collaboration et al. 2015b) and HESS J1832-093 (H. E. S. S. Collaboration et al.|2015a), respectively. The small blue circle indicates the location of $1720 \mathrm{MHz} \mathrm{OH}$ maser (Frail et al. 2013). 

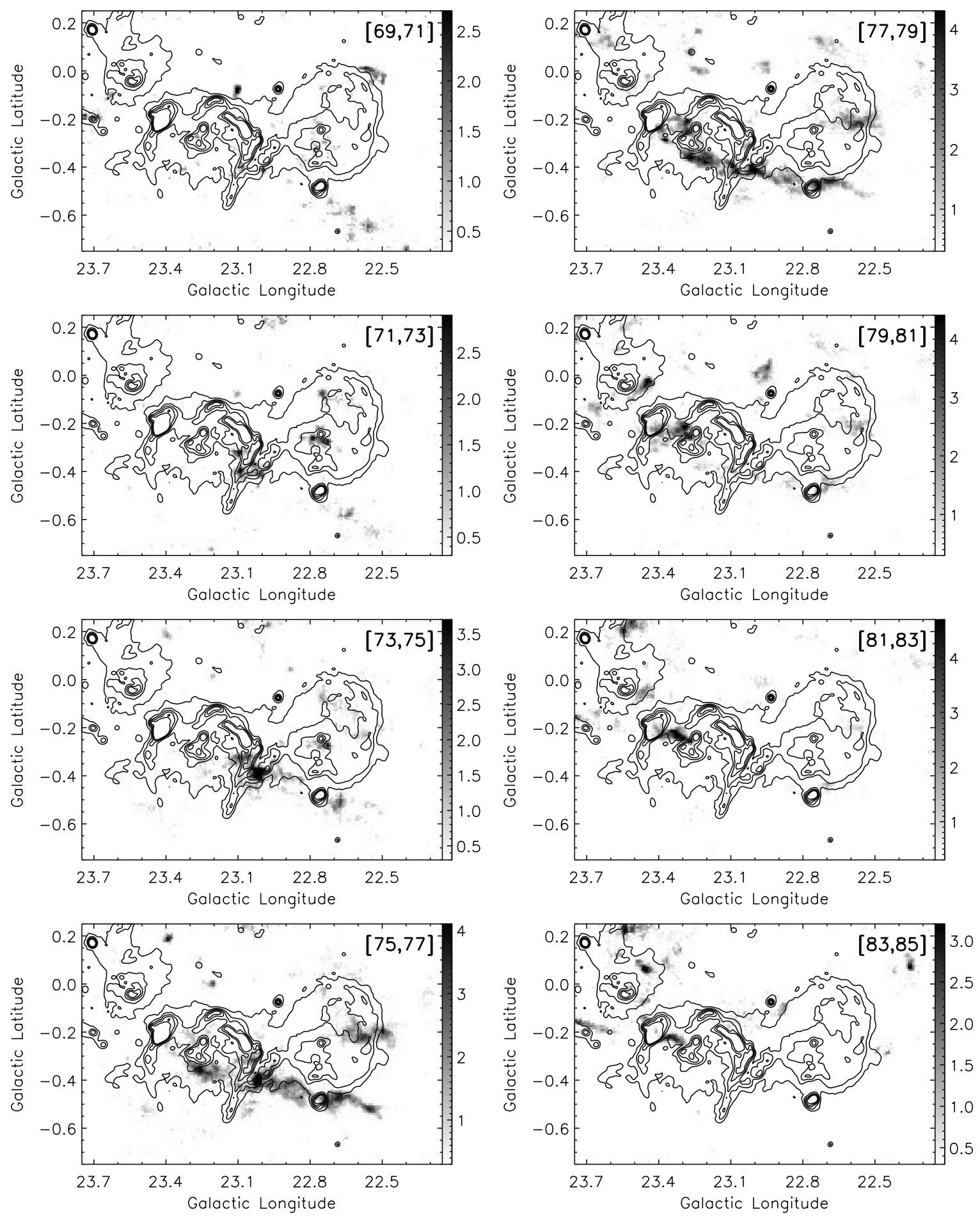

Fig. 5.- Velocity channel maps of the $\mathrm{C}^{18} \mathrm{O}(J=1-0)$ emission line in units of $\mathrm{K} \mathrm{km} \mathrm{s}^{-1}$, overlaid with the VGPS $1.4 \mathrm{GHz}$ radio continuum emission contours. The velocity range used for the integration is indicated in the top-right conner of each panel. Note that the intensity scales are different from each other. 

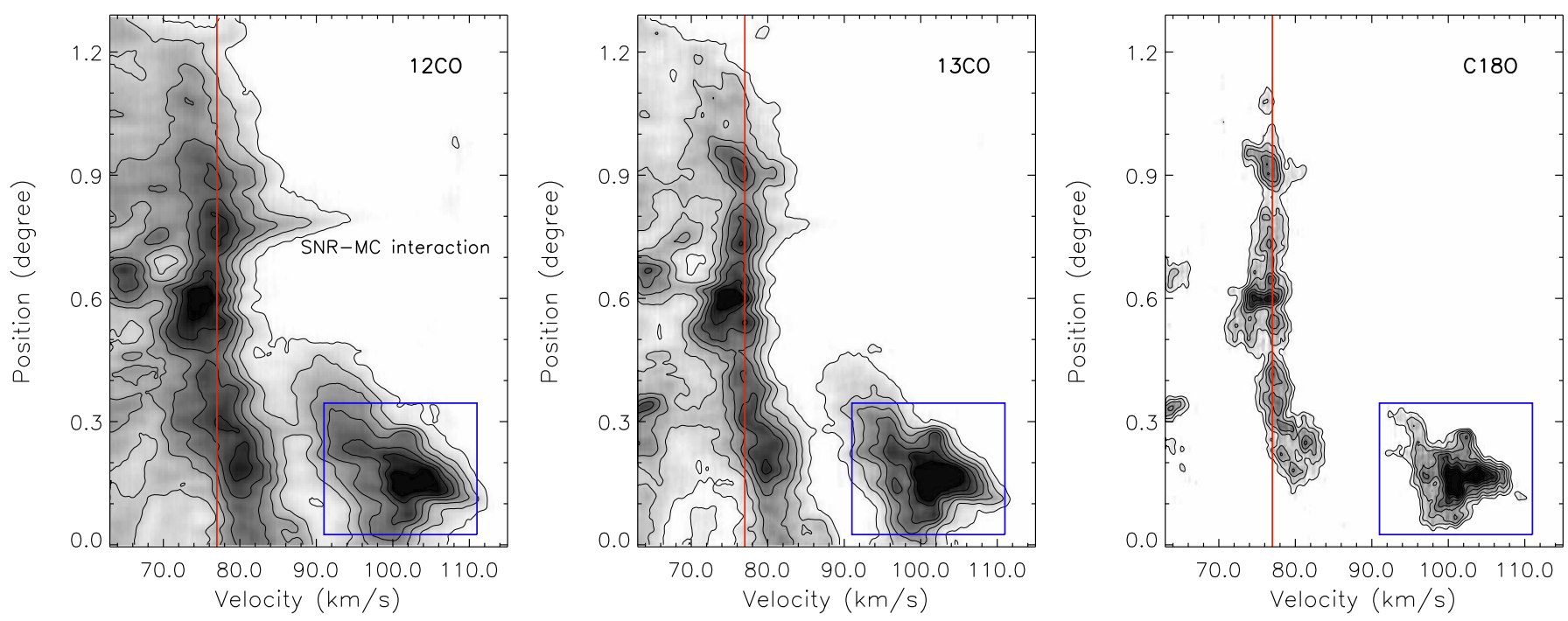

Fig. 6. - PV diagrams of the ${ }^{12} \mathrm{CO}(J=1-0),{ }^{13} \mathrm{CO}(J=1-0)$, and $\mathrm{C}^{18} \mathrm{O}(J=1-0)$ emission along the filamentary GMC G23.0-0.4 (see the long blue arrow in Figure 4). The position is measured along the long arrow $((l=23.575, b=-0.185)$ to $(l=22 \circ 370, b=-0.626))$ with a width of 10.5 . The red line marks the LSR velocity of $77 \mathrm{~km} \mathrm{~s}^{-1}$. The blue rectangle shows the G23.4 cloud near the tangent point (Ohishi et al. 2012).

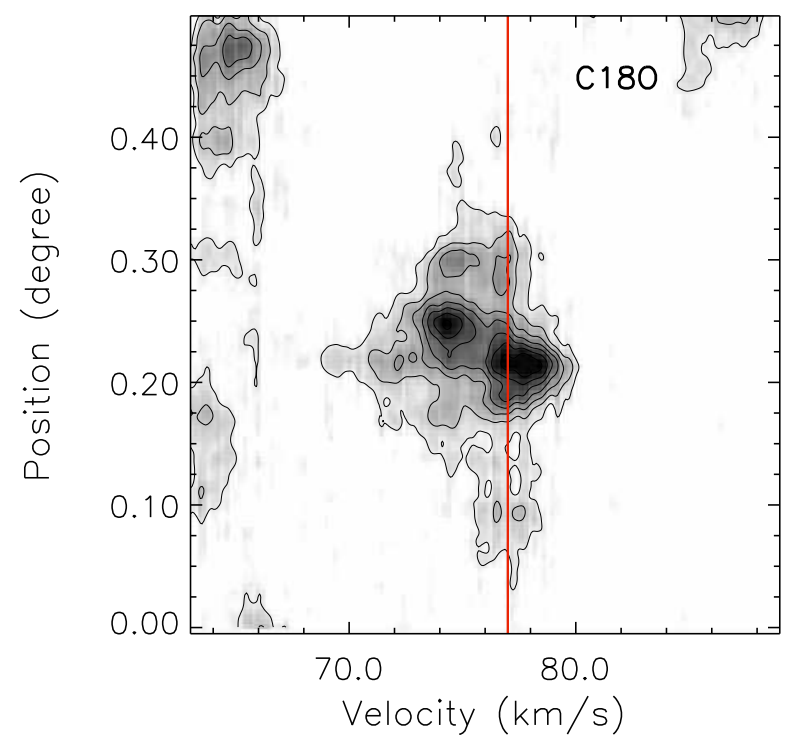

Fig. 7.- PV diagram of the $\mathrm{C}^{18} \mathrm{O}(J=1-0)$ emission along the interface between SNR W41 and SNR G22.7-0.2 (see the short blue arrow in Figure 4). The position is measured along the short arrow $\left((l=23.081, b=-0.618)\right.$ to $\left.\left(l=22^{\circ} .950, b=-0^{\circ} .127\right)\right)$ with a width of 9.5 . The red line marks the LSR velocity of $77 \mathrm{~km} \mathrm{~s}^{-1}$. 

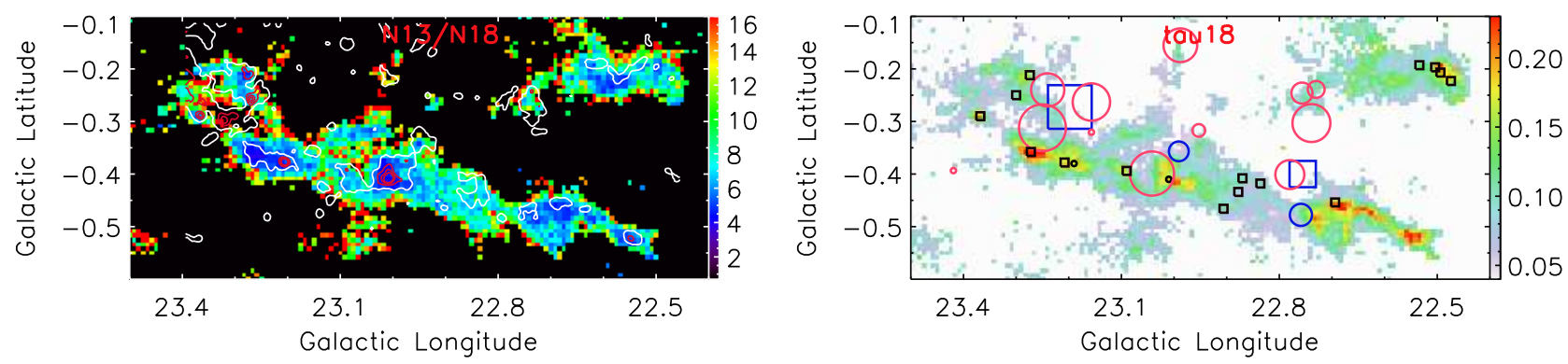

Fig. 8. - Left panel: Map of the abundance ratio $\mathrm{X}_{13 \mathrm{CO}} / \mathrm{X}_{\mathrm{C}^{18} \mathrm{O}}$ overlaid with the ATLASGAL $870 \mu \mathrm{m}$ continuum emission contours $(0.2,1.2,2.2$, and $3.2 \mathrm{Jy} / \mathrm{beam})$. The dense dust clumps $(>1.2 \mathrm{Jy} /$ beam) are highlighted in red contours. Right panel: Map of the optical depth of the $\mathrm{C}^{18} \mathrm{O}(J=1-0)$ emission. The black boxes, black circles, red circles, blue circles, and blue boxes show the fifteen dust-continuum-identified MC clumps, the two $6.7 \mathrm{GHz}$ methanol masers, the twelve H II regions, the two H II/SNR complexes, and the two massive star groups, respectively.

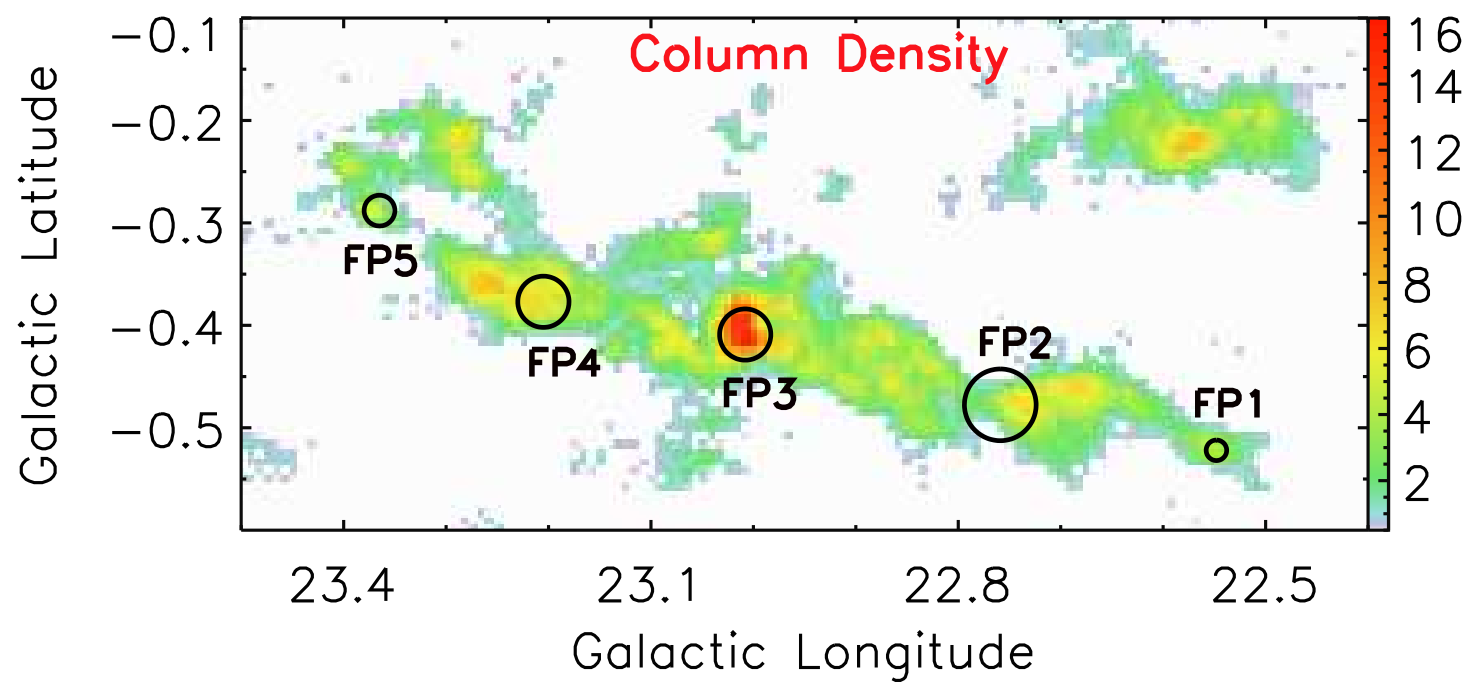

Fig. 9.- Map of the column density from $\mathrm{C}^{18} \mathrm{O}(J=1-0)$ emission with a logarithmic scale in units of $10^{22} \mathrm{~cm}^{-2}$. The four fragmentation points are marked with black circles. 